\title{
Risk Perception and Occupational Accidents among a Group of Egyptian Construction Workers in a Construction Company in Cairo.
}

\author{
Manar M. Ellaban ${ }^{1 *}$, Mervat H. $\operatorname{Rady}^{1}$, Hebat Allah MS. Gabal ${ }^{1}$, Nayera S. \\ Mostafa ${ }^{1}$. \\ ${ }^{1}$ Department of Community, Environmental and Occupational Medicine, Faculty of \\ Medicine, Ain Shams University. \\ Received: August 2018 accepted: September 2018
}

\begin{abstract}
Background: Construction sector is one of the main pillars of the global economy, even though it is a risky business in which fatal and non-fatal occupational injuries occur frequently. Information about workers' awareness about health and safety hazards, risk perception and safe work practices can help to understand where to apply prevention strategies. Unfortunately, construction sites are workplaces with limited access for research; studies at work level represent $2.28 \%$ of all available research. Objectives: To estimate the perception of construction workers to the occupational hazards, to identify types of common occupational accidents involving construction workers in an Egyptian company and to identify the workers' personal and work related characteristics determinants on their risk perception. Material and methods: A cross sectional study was conducted among construction workers in one of the projects in Cairo. During the period from January to August 2018; a sample of 104 workers were interviewed using a structured questionnaire inquiring about sociodemographic characteristics, work related variables together with assessment of workers' risk perception. Results: the majority of the workers had low risk perception for falls, contact with chemicals, struck by objects, sharp objects, manual lifting, repetitive movements and heat stress, while the noise was reported as " no or acceptable risk category". The study identified age, health and safety training, working hours and the use of PPEs as significantly related factors to risk perception. Conclusion: risk perception among studied workers in average is low. Older age, prolonged working hours, lack of training and not wearing PPEs are significant risk factors shaping workers' perception. Recommendation: safety training and change of work environment may improve workers' perception toward occupational accidents and subsequently reduce the risk of injuries.
\end{abstract}

Key words: Construction workers - Risk - Perception - Accidents- Safety.

* Corresponding author: Manar Mohamed Ibrahim Ellaban. Email:

manarellaban@med.asu.edu.eg

\section{Introduction}

Construction industry is an economic investment as construction workers represent around 180 million people, or $7 \%$ of global employment. The construction sector in Egypt is one of the main contributors to the country's economy and one of its fastest-growing sectors; it makes up around $70 \%$ of casual wage workers ${ }^{(1)}$. However, construction industry is a very hazardous industry in which fatal and non-fatal occupational injuries occur 
frequently due to the unique environment of the construction industry, human behavior, rough worksite conditions, and poor safety management ${ }^{(2)}$. The leading causes of construction workers' fatalities were falls, struck by objects, electrocution and caught-in/between. Globally, in 2016 , it was estimated that $21 \%$ of occupational fatalities recorded in construction ${ }^{(3)}$. According to a study conducted in Egypt, approximately $13 \%$ of work- related deaths and $18 \%$ of occupational injuries were recorded in construction ${ }^{(1)}$. Occupational injuries and illnesses have their impact not only on safety and health, but also the high economic impact ${ }^{(4)}$. Failure to deal with risks efficiently has been shown to cause cost and time overruns in construction projects. Risk is a calculation of the probability of the hazard to occur, and the severity of its consequences. Being able to accurately assess the risk in a situation is, at a personal level, dependent on an individual's risk perception and risk tolerance $^{(2,5)}$. Risk perception is the ability of an individual to determine a certain amount of risk, and risk tolerance refers to a person's ability to accept a certain amount of risk. Therefore, the study of workers' risk perception is important, as individuals are responsible for the risks perceived in their work environment ${ }^{(6)}$. When workers are aware of the health and safety risks in their workplace, they can follow safe work practices. Information about workers' current and changing awareness about health and safety hazards, risk perception and safe work practices can help to understand where to apply prevention strategies ${ }^{(5)}$. Unfortunately, construction sites are workplaces with limited access for research; studies at work level represent $2.28 \%$ of all available research that makes it necessary to pay more attention to safe construction environment ${ }^{(7)}$. The objectives of this study were: to estimate the perception of a group of construction workers to the hazards in their work environment, to identify the role of workers' personal and work related characteristics on their risk perception and to identify common occupational accidents' types involving construction workers in an Egyptian company.

\section{Material and methods}

Study design: A cross sectional study was conducted in one of the construction projects running in Cairo; during the period from January to August, 2018. Study population: a sample of Egyptian construction workers in a project in Cairo. Inclusion criteria: all types of construction workers in the assigned project who are working in the construction sector at least for 6 months and agreed to participate in the study. Exclusion criteria: managerial personnel, engineers and employees responsible for health and safety of staff and workplace. Sampling method: a convenience sample of construction workers at the project was recruited; the construction project site was visited 3 days a week till the required sample size was reached. Sample size: Assuming a proportion of workers who properly perceived the risk of a certain hazard to be high or permanent, a proposed $50 \%$ will yield the maximum sample size of 104 which produces a two-sided $95 \%$ confidence interval with a width equal to $20 \%(40 \%-60 \%)$ using PASS 11 program. Study tools: A structured interview questionnaire was used to collect the necessary data about workers and workplace at the construction project. The questionnaire included: Section I: socio-demographic characteristics of the studied workers e.g. (age, education, occupation, marital status, smoking habit). Section 
II: work related variables e.g. (type of contract, working hours per week, duration of work in construction and in current job, experience of injuries in the last year, wearing PPEs, receiving health and safety training, their opinion about types of the hazards in their workplace and the frequency of these hazards). Section III: assessment of workers' risk perception towards their occupational hazards using the classic Risk formula: Risk $=\mathrm{P} * \mathrm{D}$ where $\mathrm{P}$ is the probability of threat (i.e., the likelihood) and $\mathrm{D}$ is the expected damage (i.e., the severity), for quantitative risk assessment, then the risk perception was classified as: Acceptable/no risk ( 1 - 4), low risk (5 9), medium risk (10 - 15), high risk (16 $-25)^{(8,9,10,11)}$. Pilot study: a pilot study was conducted on 20 workers and the required modifications of the questionnaire were performed as adding a choice of disc prolapsed in the question of types of occupational injuries the worker has experienced and a choice of goggle and mask in the question of types of personal protective equipments used. Pilot data were excluded from the study results. Ethical Consideration: the required ethical and administrative approvals were obtained and an informed verbal consent was obtained from each participant. To assure the confidentiality, the questionnaire was anonymous. Data Management and Analysis: the collected data was revised, coded, tabulated and introduced to personal computer then analyzed using SPSS program (Statistical Package for Social Sciences) for windows Version 22. Qualitative data were presented as frequencies and percentages, while quantitative variables were presented as mean, standard deviation (SD). Chi square test and Fisher's Exact test were used and level of significance used was $\mathrm{p} \leq 0.05$.

\section{Results}

The current study included 104 workers who were working in one of the construction project in Cairo. More than half of the workers $(60.6 \%)$ were adolescents and young adults; their age ranged from 15 to 65 years with mean about $31 \pm 12$ years, nearly half of the studied workers (45.2\%) had completed secondary or technical school, $63.5 \%$ were married and $58.7 \%$ were current smokers. More than half of the current smokers $(62.3 \%)$ were smoking usually while working (Table 1). Regarding participants' work related data: $(77.9 \%)$ of the workers were working according to a part time contract, the duration of working in construction ranged from 0.5 to50 years with mean about $14 \pm 12$ years while the duration of working in current job ranged from 0.1 to 40 with mean about $6 \pm 8$ years. The working hours per week ranged from 10 hours to 72 hours with mean of $50.5 \pm 8$ hours. Regarding their occupation, the current study showed that painters represented the highest percentage of the workers (28.8\%) followed by carpenters (20.2\%) and construction laborer $(18.3 \%) \quad$ (Table 2). Concerning experience of injuries at work, 59.6\% of the studied workers had experienced injuries during the last year; of which, the most frequently encountered injuries were contusions $(48.1 \%)$ and abrasions (45.2\%) followed by fractures (37.5\%) (Table 3). Regarding the PPEs, $68.3 \%$ of the workers were wearing PPEs and the most frequently used PPEs were safety shoes (57.7\%) and the least were mask and goggle (2.9\% each) (Table 4). As regards health and safety training; $61.5 \%$ of the workers reported that they had never received training about all the reported hazards. The very frequent hazards were repetitive movements $(88.5 \%)$, noise $(87.5 \%)$, contact with chemicals $(73.1 \%)$ and heat stress $(61.5 \%)$ 
followed by manual lifting (58.7\%) and sharp objects $(58.7 \%)$. While collapse $(84.6 \%)$, fall $(76 \%)$ and struck by objects $(52.9 \%)$ were reported as quite frequent hazards. In this study, we aimed to estimate workers' risk perception through asking about the probability and severity of the studied hazards. As regards the probability, the present study showed that repetitive movements was reported by $20.2 \%$ of the studied workers as very likely to cause injuries and other hazards were reported as sometime causing injuries except for noise and heat stress were reported as never causing injuries by $(29.8 \%)$ and $(17.3 \%)$ of the workers respectively. Concerning severity of the studied hazards, the current study found that nearly two thirds of the workers reported that struck by objects, repetitive movements and noise as less severe hazards and causing injuries not requiring medical intervention, also sharp objects and manual lifting were reported as less severe hazards by $54.8 \%$ and $36.5 \%$ of the workers respectively. About half of the workers considered fall, collapse and contact with chemicals as hazards which cause injuries requiring medical intervention. However, noise, heat stress and repetitive movements are reported as not severe hazards at all as reported by $38.5 \%, 25 \%$ and $23.1 \%$ respectively. Study results revealed that the majority of the workers had low risk perception for all the hazards except for the noise as there had between no or acceptable risk perception by nearly half of the workers (Table 5). Concerning the factors affecting the level of workers' perception of different types of their occupational hazards; the present study found that there was a statistically significant relationship between age and risk perception of repetitive movements $\quad(p=0.043)$, while a statistically significant relationship was revealed between working hours per week was and manual lifting ( $\mathrm{p}=$ 0.026). Also, risk protection perception of wearing PPEs was significantly related to perception of manual lifting $(\mathrm{p}=0.0136), \quad$ repetitive movements $(\mathrm{p}=0.007)$ and heat stress $(\mathrm{p}=0.003)$. In addition, There was a statistically significant relationship between receiving health and safety training and risk perception of contact with chemical $(p=0.007)$, improper manual lifting $(\mathrm{p}=0.032)$, repetitive movements $(\mathrm{p}=0.042)$ and noise $(\mathrm{p}=0.020)$.

\section{Discussion}

Risk perceptions are important determinants of health and risk-related decisions like adopting healthy behaviors, using PPEs, and they contribute a lot in safety management, and curtailing unhealthy behaviors (12, 13). The present study revealed that more than half of the participated workers had experienced injuries in the last year $(59.6 \%)$. This rate is higher than a previous study in 2013, conducted in Egypt by Abbas et al. which revealed that $46.2 \%$ of workers had experienced Occupational injuries in the past 12 months $^{(1)}$. Also, Antonucci et al. reported that $28.3 \%$ of the workers reported their experience of at least one accident during their working life and Dong, who found that approximately $11.5 \%$ of the construction workers in Hong Kong reported they had experienced a workrelated injury during their working life ${ }^{(11,14)}$.This disagreement may be due to better safety measures, strict supervision of work environment and the use of PPEs in western countries or may be due to the younger age of the present study' workers that makes them prone to accidents. The most frequently encountered injuries among our study participants were contusions, abrasions, fractures, eye injuries, ear injuries and disc prolapse. This result nearly agrees with a previous study in

Medical Integrated Students' Research Journal (MISRJ) $\quad$ Vol. 1 No. 1 September 2018 
2012 conducted by Elsafty et al. who reported that the majority of injuries reported in Egypt were eyes injuries, shoulder and back pain, and sprain in ankles $^{(15)}$. As regards the probability of the studied hazards, the present study showed that all the studied hazards were reported as more likely to cause injuries except for noise and heat stress were reported as never causing injuries. Concerning the severity; about half of the workers considered fall as hazard which cause injuries requiring medical intervention. This result agrees with a study in 2010 conducted by Antonucci et al. determined that sharp objects, manual lifting and falling from a height were the most probable hazards causing injuries while noise was reported as unlikely hazards to cause injuries $^{(11)}$. Additionally, falling from a height was considered the most harmful hazard and causing injuries requiring medical intervention. Those findings are consistent with Elsafty et al. who reported that falls represent about $33 \%$ of all construction fatalities $^{(15)}$. When estimating the risk perception of the studied hazards among workers, the present study showed that the majority of the workers had low risk perception for all the hazards except for the noise as they had between no or acceptable risk perception by nearly half of the workers. This disagrees with a study done in Italy by Antonucci et al. in 2010 who revealed that the workers considered all the hazards as medium risk hazards ${ }^{(11)}$. This could be explained to my opinion by that the majority of the Egyptian workers may have optimism bias culturally which refers to people's perception that negative events are less likely to happen to the individual than to one's peers and is often referred to as the belief that "it won't happen to me" and lack of awareness about the consequences of these hazards as appear from lack of training by about two thirds of the workers $(61.5 \%)^{(16)}$. The current study found that four out of nine risk factors were associated with proper risk perception of different construction hazards: older age of the workers as the older workers perceived the hazard as "no or low risk category", health and safety training where the workers who received training had higher perception level, working hours where the prolonged working hours affecting the perception level negatively and the use of PPEs as the workers who were not using PPEs were found to have lower level of perception toward the studied hazards. These factors and others were reported as well by other studies conducted in various countries. In 2012, Elsafty et al. reported the positive impact of implementing educational programs on workers' perception of the hazards ${ }^{(15)}$. Similarly, a study performed in 2008 by Arezes and Miguel among workers in Scotland found that there was a statistical significant differences between the workers' risk perception and their age and the use of PPEs ( $\mathrm{p}<$ $0.001)^{(17)}$. In 2014, Perlman et al. also found that workers with more work experience and more formal safety training perceived the level of risk higher than those with little work experience and little formal safety training ${ }^{(18)}$. However, this disagrees with an Italian study done by Antonucci et al. in 2010 who revealed that training courses had a limited influence on the workers' perception of risks $^{(11)}$. Identifying factors related to risk perception of various construction hazards would raise an alarm to mitigate these factors as the majority of them being modifiable through proper health education messages to the participating workers and to improve their risk perception and prevent further occurrence of injuries. 


\section{Conclusions}

The study concluded that risk perception among studied workers in average is low. Older age of the workers, prolonged working hours, lack of training and not wearing PPEs are significant risk factors shaping the workers' perception.

\section{Limitations}

There was a difficulty to reach a large sample size as not all the workers will accept to respond due to the unique nature of their work. Also, large sample is in large projects and there was a difficulty to obtain the approvals to conduct the study there.

\section{Acknowledgements}

The authors would like to thank the administration of the construction project and all workers who agreed to provide valuable information.

\section{Funding}

This research did not receive any specific grant from funding agencies in the public, commercial, or not forprofit sectors.

\section{References:}

1. Abbas RM and Zalat MM: Non-Fatal Occupational Injuries and Safety Climate: A Cross-Sectional Study of Construction Building Workers. Journal of Safety Science and Technology. 2013, 3(12), 69-79.

2. El-Sayegh SM: Risk assessment and allocation in the UAE construction industry. International Journal of Project Management. 2008, 26(4), 431438.

3. Occupational Safety and Health Administration (OSHA) (2017): OSHA Data \&Statistics. Available at: https://www.osha.gov/oshstats/. Accessed in Mar. 2018

4. Pinto A, Nunes IL and Ribeiro RA: Occupational risk assessment in construction industry-Overview and reflection. Safety Science. 2011,49(49), 616-624.

5. National Safety Council: Risk awareness and perception of health and safety in the workplace.2017.Available at: https://www.nsc.org/worksafety/tools-resources/campbellinstitute. Accessed in Mar. 2018

6. Carriço A , Anilson RCand Gomes APG. Quantitative analysis of the construction industry workers' perception of risk in municipalities surrounding Salvador. Procedia Manufacturing. 2015, 3, $1846-1853$.

7. Forteza FJ, Sesé A and CarreteroGómez JM: CONSRAT. Construction sites risk assessment tool. Safety Science. 2016, 89, 338-354.

8. Chan A, Yeung J, Yu C, Wang S, Ke $\mathrm{Y}$ : Empirical study of risk assessment and allocation of public-private partnership projects in China. J. Manage. Eng. 2011, 27, 136-148.

9. Flammini F, Gaglione A, Mazzocca N, Pragliola C: Optimisation of security system design by quantitative risk assessment and genetic algorithms. Int. J. Risk Assess. Manage. 2011, 15, 205221.

10.Fleming $M$ and Lardner R: Strategies to promote safe behaviour as part of a health and safety management system, HSE Books. 2002.

11.Antonucci A, Giampaolo LDi, Zhang QL, Cipolla ES, and D'abruzzo C: Safety in construction yards: perception of occupational risk by italian building workers.European journal of inflammation. 2010, 8 (2), 107-115.

12.Loewenstein GF, Weber EU, Hsee CK and Welch N: Risk as Feelings. Safety Science. 2001, 127(2), 267-286.

13.Xia N, Wang X, Griffin MA, Wu C and Liu B: Do we see how they perceive risk? An integrated analysis of risk perception and its effect on workplace safety behavior. Accident Analysis \& Prevention. 2017, 106, 234-242.

14.Dong X: Long workhours, work scheduling and work-related injuries among construction workers in the United States. Scandinavian Journal of Work, Environment and Health, 2005, 31(5), 329-335. 
15.Elsafty A, Elsafty A and Malek M: Construction Safety and Occupational Health Education in Egypt, the EU, and US Firms. Open Journal of Civil Engineering. 2012, 2(9), 174-182.

16.Caponecchia C and Sheils I:Perceptions of personal vulnerability to workplace hazards in the Australian construction industry. Journal of Safety
Research. 2011, 42(4), 253-258.

17.Arezes PM and Miguel AS: Hearing protection use in industry: The role of risk perception: Safety Science. 2005, 43, 253-267.

18.Perlman, A., Sacks, R., and Barak, R. Hazard recognition and risk perception in construction. Safety Science. 2014, 64, 22-31. 
Table (1): Characteristics of the studied workers $(n=104)$

\begin{tabular}{|c|c|c|}
\hline Characteristics & & No. (\%) \\
\hline \multirow{4}{*}{ Age } & $15-$ & $63(60.6)$ \\
\hline & $30-$ & $22(21.2)$ \\
\hline & $45-$ & 15 (14.4) \\
\hline & $>=60$ & $4(3.8)$ \\
\hline \multirow{6}{*}{ Education } & Illiterate & $13(12.5)$ \\
\hline & Read and write & $12(11.5)$ \\
\hline & Primary & $3(2.9)$ \\
\hline & Preparatory & $15(14.4)$ \\
\hline & Secondary/technical & $47(45.2)$ \\
\hline & university education & $14(13.5)$ \\
\hline \multirow{3}{*}{ Marital status } & Single & $37(35.6)$ \\
\hline & Married & $66(63.5)$ \\
\hline & Divorced & $1(0.9)$ \\
\hline \multirow{3}{*}{ Smoking habits } & Current smoker & $61(58.7)$ \\
\hline & Former smoker & $7(6.7)$ \\
\hline & Non smoker & $36(34.6)$ \\
\hline \multirow{3}{*}{$\begin{array}{l}\text { Smoking while working(no. } \\
\text { smokers=61) }\end{array}$} & Usually & $38(62.3)$ \\
\hline & Sometimes & $11(18.0)$ \\
\hline & Never & $12(19.7)$ \\
\hline
\end{tabular}

Table (2): Work related characteristics of the studied workers $(n=104)$

\begin{tabular}{|l|l|l|l|}
\hline Work related characteristics & No. & \% \\
\hline \multirow{3}{*}{ Type of contract } & Full time & 23 & 22.1 \\
\cline { 2 - 4 } & Part time & 81 & 77.9 \\
\hline \multirow{4}{*}{ Job title } & Painter & 30 & 28.8 \\
\cline { 2 - 4 } & Carpenter & 21 & 20.2 \\
\cline { 2 - 4 } & Laborer & 19 & 18.3 \\
\cline { 2 - 4 } & Supervisor & 14 & 13.5 \\
\cline { 2 - 4 } & Builder & 10 & 9.6 \\
\cline { 2 - 4 } & Iron worker & 5 & 4.8 \\
\cline { 2 - 4 } & Demolition worker & 5 & 4.8 \\
\hline & Range( min-max) & Median & Mean \pm SD \\
\hline Working hours per week & $62(10-72)$ & 48 & $50.5 \pm 8.4$ \\
\hline $\begin{array}{l}\text { Duration of working in construction } \\
\text { in years }\end{array}$ & $49.5(0.5-50)$ & 10 & $14.2 \pm 12.2$ \\
\hline Duration of current job in years & $39.9(0.1-40)$ & 2 & $5.8 \pm 8$ \\
\hline & & & \\
\hline
\end{tabular}


Table (3): Experience of work related injuries among the studied workers $(n=104)$

\begin{tabular}{|l|l|l|}
\hline Work related injuries characteristics & No. $(\%)$ \\
\hline Experience of injuries during last year & $62(59.6)$ \\
\hline \multirow{4}{*}{ Type of injuries* } & Contusions & $50(48.1)$ \\
\cline { 2 - 3 } & Abrasion & $47(45.2)$ \\
\cline { 2 - 3 } & Fractures & $39(37.5)$ \\
\cline { 2 - 3 } & Eye and ear injuries & $36(34.6)$ \\
\cline { 2 - 3 } & Disc prolapsed & $4(3.8)$ \\
\hline Medical intervention & & $23(37.1)$ \\
\hline Hospital admission & Mean \pm SD & $4(6.5)$ \\
\hline \multirow{2}{*}{ Work days missed } & Min - Max & $17.9 \pm 65.7$ \\
\hline
\end{tabular}

* More than one injury could be confronted

Table (4): PPEs used by the studied workers $(n=104)$

\begin{tabular}{|l|l|l|}
\hline PPES & No. (\%) \\
\hline Use of protective equipment & $71(68.3)$ \\
\hline \multirow{4}{*}{ Type of PPEs used } & Safety shoes & $60(57.7)$ \\
\hline & Gloves & $39(37.5)$ \\
\cline { 2 - 3 } & Vest & $31(29.8)$ \\
\cline { 2 - 3 } & Helmet & $30(28.8)$ \\
\cline { 2 - 3 } & Back strap & $8(7.7)$ \\
\cline { 2 - 3 } & Google & $3(2.9)$ \\
\cline { 2 - 3 } & Mask & $3(2.9)$ \\
\hline
\end{tabular}

Table (5): Studied workers' risk perception towards the studied hazards $(n=104)$

\begin{tabular}{|l|l|l|l|l|}
\hline \multicolumn{2}{|l}{} & \multicolumn{3}{l}{} \\
\hline & $\begin{array}{l}\text { No or acceptable } \\
\text { risk }\end{array}$ & Low risk & $\begin{array}{l}\text { Medium } \\
\text { risk }\end{array}$ & $\begin{array}{l}\text { High } \\
\text { risk }\end{array}$ \\
\hline Hazards & No. (\%) & No. (\%) & No. (\%) & No. (\%) \\
\hline Fall & $4(3.9)$ & $46(45.1)$ & $39(38.2)$ & $13(12.7)$ \\
\hline Struck by object & $27(26.0)$ & $60(57.7)$ & $16(15.4)$ & $1(1.0)$ \\
\hline Repetitive movement & $34(32.7)$ & $40(38.5)$ & $28(26.9)$ & $2(1.9)$ \\
\hline Contact with chemicals & $31(30.1)$ & $72(69.9)$ & $0(0.0)$ & $0(0.0)$ \\
\hline Collapse & $5(4.9)$ & $57(55.3)$ & $29(28.2)$ & $12(11.7)$ \\
\hline Manual lifting & $23(22.1)$ & $53(51.0)$ & $23(22.1)$ & $5(4.8)$ \\
\hline Sharp objects & $14(13.6)$ & $57(55.3)$ & $32(31.1)$ & $0(0.0)$ \\
\hline Noise & $51(49.0)$ & $40(38.5)$ & $13(12.5)$ & $0(0.0)$ \\
\hline Heat & $39(37.5)$ & $50(48.1)$ & $14(13.5)$ & $1(1.0)$ \\
\hline
\end{tabular}

